\title{
Examining Changes to Food and Beverage Availability and Marketing in a Low-Income Community after the Opening of a New Supermarket: A Quasi-Experimental Study
}

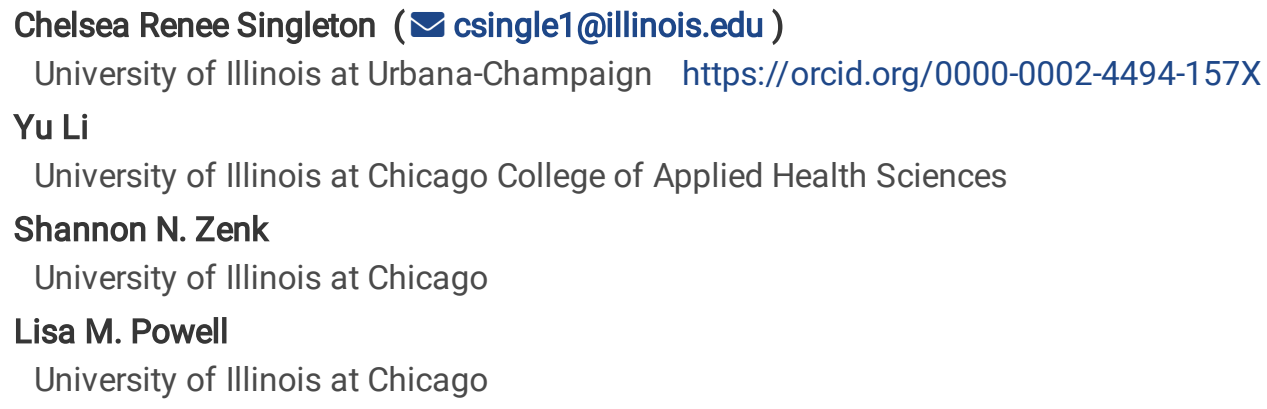

\section{Research article}

Keywords: Healthy Food Financing Initiative; Food Access; Food Marketing; Supermarket; Low-Income; African American.

Posted Date: July 27th, 2020

DOI: https://doi.org/10.21203/rs.3.rs-44242/v1

License: @ (i) This work is licensed under a Creative Commons Attribution 4.0 International License. Read Full License 


\section{Abstract}

Background: In 2016, a large chain supermarket opened in the Englewood community of Chicago, IL - a low-income African American community. The development of this supermarket was supported, in part, by the Healthy Food Financing Initiative (HFFI) - a federal initiative to expand access to healthy foods in food deserts. The aim of this study was to examine changes to food and beverage availability and marketing in Englewood's existing food stores after the supermarket's opening.

Methods: A quasi-experimental study was conducted from 2016 - 2018. Trained fieldworkers audited all small grocery and limitedservice stores (e.g., convenience stores, liquor stores, pharmacies, dollar/discount stores) located within one-square mile of the new supermarket and a one-square mile area of a demographically comparable community in Chicago that also lacked a supermarket. All stores in the one-square mile area were audited at three time points: before (2016) and after (2017 and 2018) the supermarket opened. Extensive data on availability and marketing were collected for staple food items, snacks, and beverages. Difference-indifferences (DID) regression models were used to identify significant differences between the intervention and comparison communities in the changes in food and beverage availability and marketing.

Results: Of the 78 stores audited at baseline, $71.8 \%$ were limited-service stores, and $85.9 \%$ accepted Supplemental Nutrition Assistance Program (SNAP) benefits. The availability of healthy food and beverage options in existing food stores was limited at baseline and both follow-up periods. Stores in the intervention community offered, on average, $<3$ fresh vegetable options and $<2$ fresh fruit options at all three time periods. DID regression models revealed a significant increase in 1) the percentage of stores in the intervention community offering regular cheese and promoting salty snacks at check-out from $2016-2017$ and 2) the percentage of stores in the comparison community with interior store promotions for other sweetened beverages from $2016-2018$.

Conclusions: Minimal changes in food and beverage availability and marketing occurred one and two years after the opening of the new supermarket. However, the wide range of staple food items offered by the supermarket expanded healthy food retail in Chicago's Englewood community.

\section{Background}

Increasing access to healthy foods and beverages in low-resourced communities continues to be a key public health priority in the U.S. [1-3]. Federal and state policies aimed at expanding healthy food retail provide important resources to communities that have nutritionally-vulnerable populations [4,5]. The Healthy Food Financing Initiative (HFFI) is a federally-funded program that provides grants and technical assistance to local grocers, real estate developers, and community funds interested in opening businesses that sell healthy food in communities designated as a food desert by the United States Department of Agriculture (USDA) [6-10]. Since its inception, the HFFI has supported over 1,000 projects in 35 states, which contributed to the development of several thousand square-miles of healthy food retail space in low-resourced urban and rural communities [9].

The Chicago Community Loan Fund (CCLF), a real estate investment group, received funding from the HFFI to develop a new supermarket in the Englewood community of Chicago, IL [11, 12]. Englewood is a predominately low-income and African American community located on the city's south side that lacked a large chain supermarket for over a decade [12]. Prior studies have shown that small food stores, such as convenience stores and dollar stores, can become a key food source if a community lacks a fullservice grocer [13-15]. Whole Foods Market@ agreed to partner with CCLF on the project in Englewood, so in September 2016 a new Whole Foods Market@ opened for business. Given the addition of a new supermarket to the community's food environment, it is plausible that existing small foods stores may significantly alter their food and beverage stocking and marketing practices to adjust to the competition.

The overarching aim of this research is to determine how introducing a large chain supermarket affects food and beverage availability and marketing in existing small food stores in Chicago's Englewood community at one and two years after the opening. This research contributes important knowledge to the field on 1) the environmental and economic implications of healthy food access initiatives such as the HFFI and 2) how introducing a large chain supermarket to a low-income/low-resourced community impacts the food landscape. Prior economic and public health literature on this subject is mixed [16-20]. While some studies have observed minimal changes to the types of foods and beverages stocked and marketed by existing food stores after a new 
supermarket enters $[19,20]$, others have reported significant changes to the local retail environment (i.e., store closures and sales loss) after the introduction of a mass merchandiser [16-18]. A key limitation of these studies was lack of data collection past one year after the supermarket's opening $[18,19]$. This study will address this limitation by conducting a detailed assessment of change in healthy and unhealthy food and beverage availability and marketing at one and two years after the supermarket's opening in the Englewood community.

\section{Methods}

To address the study aim, a quasi-experimental study was conducted from 2016-2018. All small non-chain grocery stores and limited-service stores (i.e., convenience stores, gas stations, dollar stores, pharmacies, and liquor stores) were mapped within a onemile radius of the new supermarket's location in Englewood. A one-square mile area of North Lawndale, a demographically similar community in Chicago, was also mapped. The selected area of North Lawndale also lacked a supermarket at the time of this study. At baseline, 96\% African American, and 49\% had an income below the federal poverty line. North Lawndale had $95 \%$ African American residents and $47 \%$ residents below poverty. Trained fieldworkers audited food and beverage availability and marketing in all food stores at three time points: 2016 (before the store opened or "baseline"), 2017 (one year after opening), and 2018 (two years after opening). Furthermore, fieldworkers audited the new supermarket at one and two years after its opening. The institutional review board at the University of Illinois Chicago considered this to be exempt research.

\section{Data Collection Tool}

Fieldworkers were trained to use the Illinois Prevention Research Center's (PRC) Food Store Audit Tool to collect data [21]. Detailed information about the tool can be found elsewhere [22]. To briefly summarize, the Illinois PRC Food Store Audit Tool is a valid and reliable instrument for assessing food and beverage availability, in-store marketing, and exterior store marketing. Over 600 variables are included on the tool including general store features, beverages, staple food items (e.g., fruit, vegetables, bread, meat, dairy), and snack foods (e.g., chips, candy, sweet backed goods). The tool is publicly available on the University of Illinois Chicago Policy, Practice and Prevention Research Center website [21]. Fieldworkers scanned the interior and exterior of each store to complete all sections of the tool. They hand-recorded their responses, and completed forms were checked by study staff for quality assurance.

\section{Variables}

\section{Store Features}

Fieldworkers assessed several store features including store type, Supplemental Nutrition Assistance Program (SNAP) authorization status, number of cash registers, service counter availability, on-site parking availability, and security feature availability. Store type was classified as small grocery store, convenience store (which includes gas stations), dollar store, pharmacy, or liquor store. The latter four store types were categorized as a limited-service store because they sold a limited line of staple food items (e.g., fruit, vegetables, bread, meat, dairy). Stores that sold a full line of staple food items, including fresh meat, were classified as a small grocery store. Convenience stores were limited-service stores that had 1-2 cash registers, but did not sell fresh meat. Limited-service stores that also sold non-food items such as household products, clothing, decorations, and small furniture were considered a dollar store. Pharmacies had a counter where customers could obtain prescription medications. Liquor stores had a store inventory that comprised $50 \%$ or more of alcoholic beverage items. Fieldworkers were asked to count and record the number of cash registers. Cash registers that were not in use or located at self-service stations counted towards the total, whereas registers located at the pharmacy, makeup, or photo counter did not count. Stores that had a butcher, bakery, or deli service counter were considered to have a service counter available. Stores that had a security guard, security camera, or security mirror were considered to have security features

\section{Food and Beverage Availability}

Fieldworkers recorded availability information for a wide range of foods and beverages from all small food stores. They counted the number of fruit and vegetable options, separately, by presentation: fresh, frozen, and canned/self-stable. An option refers to a specific type of fruit or vegetable item; for example, fresh apples, fresh oranges, and fresh pineapple were all considered separate options. Different forms of a specific food type were considered one option. For example, whole fresh apples and fresh prepackaged apple slices counted towards one option. Fieldworkers stopped counting fresh fruit and vegetable options after reaching 
20 options (recorded as $20+$ options). They stop counting frozen options and canned/shelf-stable options after surpassing 10 options (recorded as $10+$ options).

In addition to fruits and vegetables, data on the availability of the following food and beverages were also collected: milk, soda, juice, bread, cereal, rice, ground beef, cheese, and chips. For each of these items, fieldworkers scanned the store to locate a "regular" and "reduced-fat/reduced-calorie/reduced added sugar" option. If the option was available, fieldworkers recorded yes on the data collection tool. The complete list of items assessed includes regular milk (whole or $2 \%$ ), low-fat milk ( $1 \%$ or skim), regular soda, diet soda, $100 \%$ juice, juice drink ( $<50 \%$ juice), white bread, $100 \%$ whole wheat bread, regular cereal ( $\geq 6$ grams of sugar per serving), low sugar cereal ( $<6$ grams of sugar per serving), white rice, brown rice, regular ground beef ( $>10 \%$ fat), lean ground beef ( $\leq 10 \%$ fat), regular cheese, reduced fat cheese, regular chips, and low-fat chips ( 4 grams of fat per serving). Only unflavored cow's milk was assessed; plant-based milk and milk from other animals were not evaluated. Regular soda was considered any full-calorie soda (regardless of flavor) while reduced-calorie and calorie-free sodas counted towards diet soda. To be considered $100 \%$ juice or $100 \%$ whole wheat bread, the product packaging for the item had to have that exact wording. Cheese (any type) that was labeled reducedfat or part-skim was considered to be reduced-fat cheese.

\section{Food and Beverage Marketing}

Food and beverage marketing was measured by the presence of 1) interior-store item displays, 2) check-out lane displays, and 3) exterior-store advertisements. Fieldworkers scanned each food store and recorded yes or no if they found an interior-store item display for the following food and beverage items: fresh fruit, fresh vegetables, regular soda, diet soda, plain water, unflavored cow's milk, other sweetened beverages, salty snacks, sweetened baked goods, and candy. Both special floor displays and end-aisle displays were counted as interior-store item displays. Stand-alone displays of a specific food or beverage that typically force customers to walk around them were counted as a special floor display. These displays include bins, coolers, stand-alone shelving, and items arranged on a table or floor pallet. End-aisle displays refer to items arranged on the endcap of a store aisle. Sweetened beverages other than soda (e.g., juice drinks, lemonade, ready-to-drink tea, and ready-to-drink coffee) were considered other sweetened beverages. Items such as potato chips, pretzels, crackers, popcorn, and jerky were labeled salty snacks. Sweetened baked goods were items such as cookies, muffins, and snack cakes.

Fieldworkers examined the check-out lane space and recorded yes or no if they found any of the food and beverage items listed in the special floor display battery (see list above). Both self-service items and items found behind the counter, or in a display case, that required retrieval by the clerk were counted. Fieldworkers were trained to separately count the number of exterior-store advertisements found on the building and property. Counts were recorded for the following items: fresh fruits and vegetables, regular soda, diet soda, energy drinks, sports drinks, and unflavored cow's milk. Property ads were defined as advertisements found on the gates, fencing, gas pumps, or in the parking lot of the store. Fieldworkers did not count advertisements that were smaller than an $8 \times 10$ sheet of paper; they stopped counting after reaching 20 advertisement (recorded as $20+$ ads).

\section{Statistical Analysis}

Descriptive statistics (i.e., means and frequencies) were calculated to assess baseline store features by store type (small grocery vs. limited-service) and community type (intervention vs. comparison). Significant differences in the baseline features of small food stores were identified using differences in proportions and Student's $t$ tests. Means and frequencies were also calculated, by community type, for all food and beverage availability and marketing variables at each time period: 2016, 2017, and 2018. Furthermore, food and beverage availability and marketing variables were summarized and presented for the supermarket. Only small food stores found during data collection were audited each year; thus, sample sizes of stores vary by year in both communities. In the intervention community, 40,32, and 31 stores were audited in 2016, 2017, and 2018, respectively. Approximately, 38, 42, and 40 stores in the comparison community were audited in 2016, 2017, and 2018, respectively. Difference-indifferences (DID) regression models were used to identify significant differences between the intervention and comparison communities in regards to change in store food and beverage availability and marketing from baseline and post-opening of the new supermarket. DID models compared baseline data to 2017 and 2018 data, separately. DID models were adjusted for food store type and did not include data collected from the supermarket. All analyses were performed with STATA 14 and p-values $<0.05$ were considered statistically significant.

\section{Results}


Table 1 displays the baseline features of the small food stores across all stores and by store type and intervention status. Half of all stores in the baseline sample were convenience stores and $85.9 \%$ accepted SNAP benefits. Approximately $19.2 \%$ were sold gasoline and $96.2 \%$ had at least one security feature. All small grocery stores accepted SNAP whereas $80.4 \%$ of limited service stores accepted SNAP. A significantly greater percentage of small grocery stores had a service counter available. Stores located in the intervention and comparison communities had similar store features at baseline.

Table 1

- Baseline Features of Small Food Stores Stratified by Store Type and Site (2016), \% or Mean (SD).

\begin{tabular}{|c|c|c|c|c|c|}
\hline Store Feature: & $\begin{array}{l}\text { All Stores } \\
N=78\end{array}$ & $\begin{array}{l}\text { Small Grocery } \\
\mathrm{N}=22\end{array}$ & $\begin{array}{l}\text { Limited Service } \\
N=56\end{array}$ & $\begin{array}{l}\text { Intervention } \\
\mathrm{N}=40\end{array}$ & $\begin{array}{l}\text { Comparison } \\
\mathrm{N}=38\end{array}$ \\
\hline \multicolumn{6}{|l|}{ Store Type: } \\
\hline Small Grocery & $28.2 \%$ & $100 \%$ & $n / a$ & $35.0 \%$ & $21.1 \%$ \\
\hline Convenience Store & $50.0 \%$ & $n / a$ & $69.6 \%$ & $47.5 \%$ & $52.6 \%$ \\
\hline Other ${ }^{\mathrm{a}}$ & $21.8 \%$ & $n / a$ & $30.4 \%$ & $17.5 \%$ & $26.3 \%$ \\
\hline \multicolumn{6}{|l|}{ Store Features: } \\
\hline Accepts SNAP Benefits & $85.9 \%$ & $100 \%$ & $80.4 \%$ * & $85.0 \%$ & $86.8 \%$ \\
\hline Number of Cash Registers & $1.7(0.9)$ & $1.6(0.7)$ & $1.7(1.0)$ & $1.7(0.8)$ & $1.7(1.0)$ \\
\hline Butcher, Bakery, or Deli Available & $26.9 \%$ & $63.6 \%$ & $12.5 \%$ * & $27.5 \%$ & $26.3 \%$ \\
\hline Sells Gasoline & $19.2 \%$ & $0 \%$ & $26.8 \%$ * & $25.0 \%$ & $13.2 \%$ \\
\hline Parking On Site & $38.5 \%$ & $22.7 \%$ & $44.6 \%$ & $40.0 \%$ & $36.8 \%$ \\
\hline Security Guard, Cameras, or Mirror Available & $96.2 \%$ & $100 \%$ & $94.6 \%$ & $95.0 \%$ & $97.4 \%$ \\
\hline \multicolumn{6}{|c|}{ SNAP: Supplemental Nutrition Assistance Program. } \\
\hline \multicolumn{6}{|c|}{ a.) Other limited service stores include pharmacies, liquor stores, and discount/dollar stores. } \\
\hline
\end{tabular}

Information on food and beverage availability in the new supermarket and small food stores at baseline (2016) and follow-up (2017 and 2018) are recorded in Table 2. The supermarket carried $20+$ fresh and $10+$ frozen fruit and vegetable options at both follow-up periods. Small food stores in the intervention and comparison communities, on average, offered $<3$ fresh and $<2.5$ frozen fruit and vegetable options in every year of data collection. Shelf-stable fruit and vegetable options were more prevalent in small food stores compared to fresh options. Results from DID models suggested no significant differences for change in fruit and vegetable availability between stores in the intervention community and the comparison community at one and two years after the supermarket opened. The supermarket carried every food and beverage item on the data collection tool except regular ground beef. A low percentage of small food stores in both communities stocked the following healthier alternative items in every year of data collection: skim or $1 \%$ milk (2.5-6.5\%), $100 \%$ whole wheat bread $(2.5-38.7 \%)$, brown rice $(6.3-22.5 \%)$, lean ground beef $(0-6.5 \%)$, and low-fat chips (2.4-13.3\%). DID analysis detected a significant difference between stores in the intervention community and comparison community for change in regular cheese availability from 2016 to 2017 ( $\beta=4.67$; SE = 1.59). While the percentage of stores in the intervention community that offer regular cheese slightly increased from 2016 to 2017 (55.3\% vs. 62.5\%), the percentage of stores in the comparison community that offer regular cheese declined ( $52.6 \%$ vs. $31.0 \%)$. This difference was not observed in 2018. 
Table 2

- Change in Food and Beverage Availability in Small Food Stores, \% or Mean $( \pm$ SD).

\begin{tabular}{|c|c|c|c|c|c|c|c|c|c|}
\hline \multirow[t]{3}{*}{ Item: } & \multirow{2}{*}{\multicolumn{2}{|c|}{ Supermarket }} & \multicolumn{3}{|c|}{$\begin{array}{l}\text { Small Food Stores } \\
\text { Intervention Site }\end{array}$} & \multicolumn{3}{|c|}{$\begin{array}{l}\text { Small Food Stores } \\
\text { Comparison Site }\end{array}$} & \multirow{2}{*}{$\begin{array}{l}\text { DID Regress } \\
\text { Model } \\
\beta \text { (SE) }^{a} \\
\text { Follow-Up }\end{array}$} \\
\hline & & & Baseline & Follon & & Baseline & Follon & & \\
\hline & 2017 & 2018 & $\begin{array}{l}2016 \\
(N=40)\end{array}$ & $\begin{array}{l}2017 \\
(\mathrm{~N}= \\
32)\end{array}$ & $\begin{array}{l}2018 \\
(\mathrm{~N}= \\
31)\end{array}$ & $\begin{array}{l}2016 \\
(N=38)\end{array}$ & $\begin{array}{l}2017 \\
\left(\begin{array}{l}N= \\
42)\end{array}\right.\end{array}$ & $\begin{array}{l}2018 \\
(N= \\
40)\end{array}$ & 20172018 \\
\hline
\end{tabular}

\section{Fresh Produce:}

\begin{tabular}{|c|c|c|c|c|c|c|c|c|c|}
\hline Fruit Options & 20 & 20 & $1.5(2.2)$ & $\begin{array}{l}1.2 \\
(2.7)\end{array}$ & $\begin{array}{l}1.8 \\
(3.9)\end{array}$ & $1.3(2.9)$ & $\begin{array}{l}1.3 \\
(2.7)\end{array}$ & $\begin{array}{l}1.7 \\
(3.1)\end{array}$ & $n / a^{b}$ \\
\hline $\begin{array}{l}\text { Vegetable } \\
\text { Options }\end{array}$ & 20 & 20 & $2.8(3.5)$ & $\begin{array}{l}2.1 \\
(3.6)\end{array}$ & $\begin{array}{l}2.3 \\
(4.4)\end{array}$ & $1.9(3.0)$ & $\begin{array}{l}1.7 \\
(2.9)\end{array}$ & $\begin{array}{l}2.1 \\
(3.1)\end{array}$ & $\mathrm{n} / \mathrm{a}$ \\
\hline
\end{tabular}

\section{Frozen Produce:}

\begin{tabular}{|c|c|c|c|c|c|c|c|c|c|}
\hline Fruit Options & 10 & 10 & $0.1(0.3)$ & $\begin{array}{l}0.0 \\
(0.2)\end{array}$ & $\begin{array}{l}0.2 \\
(0.9)\end{array}$ & $0(0)$ & $\begin{array}{l}0.1 \\
(0.3)\end{array}$ & $\begin{array}{l}0.0 \\
(0.2)\end{array}$ & $\mathrm{n} / \mathrm{a}$ \\
\hline $\begin{array}{l}\text { Vegetable } \\
\text { Options }\end{array}$ & 10 & 10 & $2.2(2.9)$ & $\begin{array}{l}2.0 \\
(2.9)\end{array}$ & $\begin{array}{l}2.2 \\
(3.4)\end{array}$ & $1.8(2.8)$ & $\begin{array}{l}2.0 \\
(3.0)\end{array}$ & $\begin{array}{l}1.6 \\
(2.5)\end{array}$ & $\mathrm{n} / \mathrm{a}$ \\
\hline
\end{tabular}

\section{Canned Produce:}

\begin{tabular}{|c|c|c|c|c|c|c|c|c|c|c|}
\hline Fruit Options & 7 & 10 & $2.8(1.8)$ & $\begin{array}{l}2.5 \\
(1.8)\end{array}$ & $\begin{array}{l}3.0 \\
(2.1)\end{array}$ & $2.9(2.1)$ & $\begin{array}{l}2.8 \\
(2.1)\end{array}$ & $\begin{array}{l}2.8 \\
(1.9)\end{array}$ & $\begin{array}{l}-.14 \\
(.15)\end{array}$ & $.06(.18)$ \\
\hline $\begin{array}{l}\text { Vegetable } \\
\text { Options }\end{array}$ & 10 & 10 & $6.7(2.9)$ & $\begin{array}{l}6.7 \\
(2.9)\end{array}$ & $\begin{array}{l}7.3 \\
(2.9)\end{array}$ & $5.8(2.8)$ & $\begin{array}{l}6.8 \\
(3.0)\end{array}$ & $\begin{array}{l}6.2 \\
(3.0)\end{array}$ & $\mathrm{n} / \mathrm{a}$ & $\mathrm{n} / \mathrm{a}$ \\
\hline
\end{tabular}

Milk:

\begin{tabular}{lllllllllll} 
Skim or $1 \%$ & $100 \%$ & $100 \%$ & $2.5 \%$ & $6.3 \%$ & $6.5 \%$ & $5.3 \%$ & $4.8 \%$ & $5.0 \%$ & $n / a$ & $n / a$ \\
\hline Whole or $2 \%$ & $100 \%$ & $100 \%$ & $97.5 \%$ & $93.8 \%$ & $100.0 \%$ & $81.6 \%$ & $92.9 \%$ & $90.0 \%$ & $n / a$ & $n / a$
\end{tabular}

\section{Soda:}

\begin{tabular}{|c|c|c|c|c|c|c|c|c|c|c|}
\hline Diet & $100 \%$ & $100 \%$ & $85.0 \%$ & $78.1 \%$ & $77.4 \%$ & $68.4 \%$ & $71.4 \%$ & $80.0 \%$ & $\begin{array}{l}-.88 \\
(0.90)\end{array}$ & $\begin{array}{l}-1.20 \\
(1.00)\end{array}$ \\
\hline Regular & $100 \%$ & $100 \%$ & $100.0 \%$ & $100.0 \%$ & $96.8 \%$ & $100.0 \%$ & 100.00 & $100.0 \%$ & $\mathrm{n} / \mathrm{a}$ & $\mathrm{n} / \mathrm{a}$ \\
\hline \multicolumn{11}{|l|}{ Juice: } \\
\hline $100 \%$ Fruit Juice & $100 \%$ & $100 \%$ & $80.0 \%$ & $87.5 \%$ & $83.9 \%$ & $71.1 \%$ & $100.0 \%$ & $97.5 \%$ & $\mathrm{n} / \mathrm{a}$ & $\mathrm{n} / \mathrm{a}$ \\
\hline Juice Drink & $100 \%$ & $100 \%$ & $95.0 \%$ & $100.0 \%$ & $96.8 \%$ & $100.0 \%$ & $100.0 \%$ & $100.0 \%$ & $\mathrm{n} / \mathrm{a}$ & $\mathrm{n} / \mathrm{a}$ \\
\hline \multicolumn{11}{|l|}{ Bread: } \\
\hline $\begin{array}{l}\text { 100\% Whole } \\
\text { Wheat }\end{array}$ & $100 \%$ & $100 \%$ & $37.5 \%$ & $3.1 \%$ & $38.7 \%$ & $22.9 \%$ & $4.8 \%$ & $2.5 \%$ & $\mathrm{n} / \mathrm{a}$ & $\mathrm{n} / \mathrm{a}$ \\
\hline
\end{tabular}

DID: Difference-in-Difference; SD: Standard Deviation; SE: Standard Error.

${ }^{*}$-value $<0.05 ;{ }^{* *}$ p-value $<0.01 ; * \star * p-v a l u e<0.001$

a.) Beta estimates and standard errors for intervention*time interaction term in differences-in-differences regression models.

b.) DID model unable to produce results. 


\begin{tabular}{|c|c|c|c|c|c|c|c|c|c|c|}
\hline \multirow[t]{5}{*}{ Item: } & \multirow{3}{*}{\multicolumn{2}{|c|}{ Supermarket }} & \multirow{2}{*}{\multicolumn{3}{|c|}{$\begin{array}{l}\text { Small Food Stores } \\
\text { Intervention Site }\end{array}$}} & \multirow{2}{*}{\multicolumn{3}{|c|}{$\begin{array}{l}\text { Small Food Stores } \\
\text { Comparison Site }\end{array}$}} & \multirow{2}{*}{\multicolumn{2}{|c|}{$\begin{array}{l}\text { DID Regression } \\
\text { Model }\end{array}$}} \\
\hline & & & & & & & & & & \\
\hline & & & \multirow{2}{*}{$\begin{array}{l}\text { Baseline } \\
2016\end{array}$} & \multicolumn{2}{|c|}{ Follow-Up } & \multirow{2}{*}{$\begin{array}{l}\text { Baseline } \\
2016\end{array}$} & \multicolumn{2}{|c|}{ Follow-Up } & \multicolumn{2}{|l|}{ Follow-Up } \\
\hline & 2017 & 2018 & & 2017 & 2018 & & 2017 & 2018 & 20172018 & \\
\hline & & & $(N=40)$ & $\begin{array}{l}(\mathrm{N}= \\
32)\end{array}$ & $\begin{array}{l}(\mathrm{N}= \\
31)\end{array}$ & $(\mathrm{N}=38)$ & $\begin{array}{l}(\mathrm{N}= \\
42)\end{array}$ & $\begin{array}{l}(\mathrm{N}= \\
40)\end{array}$ & & \\
\hline White & $100 \%$ & $100 \%$ & $85.0 \%$ & $87.5 \%$ & $87.1 \%$ & $81.1 \%$ & $92.9 \%$ & $82.5 \%$ & .09 (1.31) & $\begin{array}{l}-.36 \\
(1.11)\end{array}$ \\
\hline \multicolumn{11}{|l|}{ Cereal: } \\
\hline Low Sugar & $100 \%$ & $100 \%$ & $32.5 \%$ & $28.1 \%$ & $41.9 \%$ & $44.7 \%$ & $40.5 \%$ & $35.0 \%$ & $\begin{array}{l}-.21 \\
(.84)\end{array}$ & $.92(.87)$ \\
\hline Regular & $100 \%$ & $100 \%$ & $90.0 \%$ & $93.8 \%$ & $93.6 \%$ & $94.7 \%$ & $92.9 \%$ & $85.0 \%$ & $\mathrm{n} / \mathrm{a}$ & $\mathrm{n} / \mathrm{a}$ \\
\hline \multicolumn{11}{|l|}{ Rice: } \\
\hline Brown & $100 \%$ & $100 \%$ & $12.5 \%$ & $6.3 \%$ & $9.7 \%$ & $15.8 \%$ & $11.9 \%$ & $22.5 \%$ & $.68(1.73)$ & $\begin{array}{l}.02 \\
(1.46)\end{array}$ \\
\hline White & $100 \%$ & $100 \%$ & $97.5 \%$ & $84.4 \%$ & $93.6 \%$ & $92.1 \%$ & $92.9 \%$ & $92.5 \%$ & $\mathrm{n} / \mathrm{a}$ & $\mathrm{n} / \mathrm{a}$ \\
\hline \multicolumn{11}{|l|}{ Ground Beef: } \\
\hline Extra Lean & $100 \%$ & $100 \%$ & $5.0 \%$ & $3.2 \%$ & $6.5 \%$ & $5.3 \%$ & $0 \%$ & $0 \%$ & $\mathrm{n} / \mathrm{a}$ & $\mathrm{n} / \mathrm{a}$ \\
\hline Regular & $0 \%$ & $0 \%$ & $32.5 \%$ & $18.8 \%$ & $12.9 \%$ & $18.4 \%$ & $11.9 \%$ & $20.0 \%$ & $\mathrm{n} / \mathrm{a}$ & $\mathrm{n} / \mathrm{a}$ \\
\hline \multicolumn{11}{|l|}{ Cheese: } \\
\hline $\begin{array}{l}\text { Reduced or Low- } \\
\text { Fat }\end{array}$ & $100 \%$ & $100 \%$ & $15.0 \%$ & $15.6 \%$ & $25.8 \%$ & $18.4 \%$ & $16.7 \%$ & $37.5 \%$ & $.56(1.20)$ & $\begin{array}{l}-.39 \\
(1.11)\end{array}$ \\
\hline Regular & $100 \%$ & $100 \%$ & $55.3 \%$ & $62.5 \%$ & $61.3 \%$ & $52.6 \%$ & $31.0 \%$ & $57.5 \%$ & $\begin{array}{l}4.67 \\
(1.59) \star \star\end{array}$ & $\begin{array}{l}1.25 \\
(1.40)\end{array}$ \\
\hline \multicolumn{11}{|l|}{ Chips: } \\
\hline Low-Fat & $100 \%$ & $0 \%$ & $12.5 \%$ & $3.1 \%$ & $13.3 \%$ & $13.2 \%$ & $2.4 \%$ & $12.5 \%$ & $\mathrm{n} / \mathrm{a}$ & $\mathrm{n} / \mathrm{a}$ \\
\hline Regular & $100 \%$ & $100 \%$ & $100.0 \%$ & $100.0 \%$ & $100.0 \%$ & $94.7 \%$ & $97.6 \%$ & $100.0 \%$ & $\mathrm{n} / \mathrm{a}$ & $\mathrm{n} / \mathrm{a}$ \\
\hline \multicolumn{11}{|c|}{ DID: Difference-in-Difference; SD: Standard Deviation; SE: Standard Error. } \\
\hline \multicolumn{11}{|c|}{${ }^{*} \mathrm{p}$-value $<0.05 ;{ }^{* \star p}$-value $<0.01 ; * \star \star p-v a l u e<0.001$} \\
\hline a.) Beta estimates & ind stanc & ard erro & for interve & tion*time & nteraction & term in diff & rences-in- & ifferences & regression $\mathrm{m}$ & dels. \\
\hline
\end{tabular}

Table 3 displays information on food and beverage marketing in the new supermarket and small food stores in both communities at baseline and follow-up. The new supermarket had exterior store ads for fresh fruits and vegetables in 2018 . About $20 \%$ of small food stores in the intervention and comparison communities had exterior store ads for fresh fruits and vegetables in each year of data collection. Regular soda was the item found advertised outside the largest percentage of small food stores at all time periods. No differences in change in exterior store advertisements were detected between communities in 2017 or 2018. Interior store displays for variety of food and beverage items were observed in the supermarket in 2017 and 2018 including fresh fruit, plain water, salty snacks, candy, and sweetened baked goods. A low-percentage of small food stores $(<20 \%)$ in both communities had interior store displays for fresh fruits and vegetables at each time period. Over $70 \%$ of small food stores had interior store displays for salty snacks and sweetened-baked goods. A significant difference between the intervention community and comparison community was detected for change in percentage of stores with interior store displays for other sweetened beverages from 2016 to 
$2018(\beta=-2.23 ; \mathrm{SE}=1.06)$. While the percentage of stores in the intervention community with interior store displays for other sweetened beverages was about the same in 2016 vs. 2018 (37.5\% vs. 38.7\%), the percentage of stores in the comparison community with interior store displays for other sweetened beverages increased (55.3\% vs. $70.0 \%$ ). About $12-23 \%$ of small food stores had fresh fruit available at check-out each year. A large percentage of small food stores in both communities had candy, salty snacks, and sweetened baked goods available at check-out. A significant difference between communities was observed for change in percentage of stores with salty snacks available at check-out from 2016 to 2017 ( $\beta=2.50 ; S E=0.97)$. The percentage of stores with salty snacks at check-out significantly increased in the intervention community one year after the supermarket opened. 
Table 3

- Change in Food and Beverage Marketing in Small Food Stores, \%.

\begin{tabular}{|c|c|c|c|c|c|c|c|c|c|c|}
\hline \multirow[t]{4}{*}{ Item: } & \multirow{2}{*}{\multicolumn{2}{|c|}{ Supermarket }} & \multicolumn{3}{|c|}{$\begin{array}{l}\text { Small Food Stores } \\
\text { Intervention Site }\end{array}$} & \multicolumn{3}{|c|}{$\begin{array}{l}\text { Small Food Stores } \\
\text { Comparison Site }\end{array}$} & \multirow{2}{*}{\multicolumn{2}{|c|}{$\begin{array}{l}\text { DID Regression } \\
\text { Model } \\
\beta(\mathrm{SE})^{\mathrm{a}}\end{array}$}} \\
\hline & & & Baseline & Follow-l & & Baseline & Follow- & & & \\
\hline & \multirow[t]{2}{*}{2017} & \multirow[t]{2}{*}{2018} & 2016 & 2017 & 2018 & 2016 & 2017 & 2018 & \multirow{2}{*}{\multicolumn{2}{|c|}{20172018}} \\
\hline & & & $(N=40)$ & $\begin{array}{l}(\mathrm{N}= \\
32)\end{array}$ & $\begin{array}{l}(\mathrm{N}= \\
31)\end{array}$ & $(N=38)$ & $\begin{array}{l}(\mathrm{N}= \\
42)\end{array}$ & $\begin{array}{l}(\mathrm{N}= \\
40)\end{array}$ & & \\
\hline \multicolumn{11}{|l|}{ Exterior Store Ads ${ }^{b}$} \\
\hline $\begin{array}{l}\text { Fresh fruits and } \\
\text { vegetables }\end{array}$ & $0 \%$ & $100 \%$ & $20.0 \%$ & $19.4 \%$ & $22.6 \%$ & $15.8 \%$ & $23.8 \%$ & $20.0 \%$ & $\begin{array}{l}.86 \\
(1.54)\end{array}$ & $\begin{array}{l}2.01 \\
(1.73)\end{array}$ \\
\hline Regular soda & $0 \%$ & $0 \%$ & $70.0 \%$ & $64.5 \%$ & $64.5 \%$ & $57.9 \%$ & $78.6 \%$ & $57.5 \%$ & $\begin{array}{l}-2.82 \\
(1.47)\end{array}$ & $\begin{array}{l}-.10 \\
(1.36)\end{array}$ \\
\hline Diet soda & $0 \%$ & $0 \%$ & $10.0 \%$ & $3.2 \%$ & $12.9 \%$ & $5.3 \%$ & $0 \%$ & $10.0 \%$ & $\mathrm{n} / \mathrm{a}^{\mathrm{e}}$ & $\mathrm{n} / \mathrm{a}$ \\
\hline Energy drink & $0 \%$ & $0 \%$ & $35.0 \%$ & $32.3 \%$ & $29.0 \%$ & $47.4 \%$ & $40.5 \%$ & $50.0 \%$ & $\begin{array}{l}-.06 \\
(1.17)\end{array}$ & $\begin{array}{l}-.62 \\
(1.22)\end{array}$ \\
\hline Sports drink & $0 \%$ & $0 \%$ & $15.0 \%$ & $9.7 \%$ & $19.4 \%$ & $10.5 \%$ & $21.4 \%$ & $15.0 \%$ & $\begin{array}{l}-2.51 \\
(1.50)\end{array}$ & $\begin{array}{l}-.18 \\
(1.57)\end{array}$ \\
\hline Unflavored-milk & $0 \%$ & $0 \%$ & $45.0 \%$ & $35.5 \%$ & $41.9 \%$ & $32.4 \%$ & $38.1 \%$ & $45.0 \%$ & $\begin{array}{l}-2.41 \\
(1.56)\end{array}$ & $\begin{array}{l}-2.14 \\
(1.63)\end{array}$ \\
\hline \multicolumn{11}{|l|}{$\begin{array}{l}\text { Interior Store Item } \\
\text { Displayc }\end{array}$} \\
\hline Fresh fruit & $100 \%$ & $100 \%$ & $2.5 \%$ & $15.6 \%$ & $9.7 \%$ & $7.9 \%$ & $4.8 \%$ & $7.5 \%$ & $\mathrm{n} / \mathrm{a}$ & $\mathrm{n} / \mathrm{a}$ \\
\hline Fresh vegetables & $0 \%$ & $100 \%$ & $2.5 \%$ & $9.4 \%$ & $6.5 \%$ & $2.6 \%$ & $7.1 \%$ & $7.5 \%$ & $\mathrm{n} / \mathrm{a}$ & $\mathrm{n} / \mathrm{a}$ \\
\hline Unflavored-milk & $0 \%$ & $0 \%$ & $2.6 \%$ & $3.1 \%$ & $0 \%$ & $0 \%$ & $0 \%$ & $0 \%$ & $\mathrm{n} / \mathrm{a}$ & $\mathrm{n} / \mathrm{a}$ \\
\hline Plain water & $100 \%$ & $100 \%$ & $10.0 \%$ & $25.0 \%$ & $9.7 \%$ & $13.2 \%$ & $16.7 \%$ & $15.0 \%$ & $\mathrm{n} / \mathrm{a}$ & $\mathrm{n} / \mathrm{a}$ \\
\hline Regular soda & $100 \%$ & $0 \%$ & $40.0 \%$ & $53.1 \%$ & $58.1 \%$ & $39.5 \%$ & $52.4 \%$ & $72.5 \%$ & $\begin{array}{l}-.85 \\
(.98)\end{array}$ & $\begin{array}{l}-1.45 \\
(1.02)\end{array}$ \\
\hline Diet soda & $100 \%$ & $0 \%$ & $22.5 \%$ & $18.8 \%$ & $12.9 \%$ & $15.8 \%$ & $21.4 \%$ & $12.5 \%$ & $\begin{array}{l}-1.22 \\
(1.22)\end{array}$ & $\begin{array}{l}-1.71 \\
(1.30)\end{array}$ \\
\hline $\begin{array}{l}\text { Other sweetened } \\
\text { beverages }\end{array}$ & $100 \%$ & $0 \%$ & $37.5 \%$ & $46.9 \%$ & $38.7 \%$ & $55.3 \%$ & $35.7 \%$ & $70.0 \%$ & $\begin{array}{l}1.31 \\
(.86)\end{array}$ & $\begin{array}{l}-2.23 \\
(1.06)^{*}\end{array}$ \\
\hline Salty snacks & $100 \%$ & $100 \%$ & $95.0 \%$ & $100 \%$ & $87.1 \%$ & $94.7 \%$ & $90.5 \%$ & $97.5 \%$ & $\mathrm{n} / \mathrm{a}$ & $\mathrm{n} / \mathrm{a}$ \\
\hline Candy & $100 \%$ & $100 \%$ & $47.5 \%$ & $71.9 \%$ & $51.6 \%$ & $71.1 \%$ & $57.1 \%$ & $87.5 \%$ & $\mathrm{n} / \mathrm{a}$ & $\mathrm{n} / \mathrm{a}$ \\
\hline
\end{tabular}

DID: Difference-In-Difference; SE: Standard Error.

*p-value $<0.05 ; * \star p$-value $<0.01 ; * \star * p-$ value $<0.001$

a.) Beta estimates and standard errors for intervention*time interaction term in differences-in-differences regression models.

b.) Exterior store ads include ads posted on the building and the store property.

c.) Interior store item displays includes special floor displays and end-aisle displays.

d.) Available at check-out includes items that are self-serve and clerk-assisted.

e.) DID model unable to produce results. 


\begin{tabular}{|c|c|c|c|c|c|c|c|c|c|c|}
\hline \multirow[t]{5}{*}{ Item: } & \multirow{3}{*}{\multicolumn{2}{|c|}{ Supermarket }} & \multirow{2}{*}{\multicolumn{3}{|c|}{$\begin{array}{l}\text { Small Food Stores } \\
\text { Intervention Site }\end{array}$}} & \multirow{2}{*}{\multicolumn{3}{|c|}{$\begin{array}{l}\text { Small Food Stores } \\
\text { Comparison Site }\end{array}$}} & \multirow{2}{*}{\multicolumn{2}{|c|}{$\begin{array}{l}\text { DID Regression } \\
\text { Model }\end{array}$}} \\
\hline & & & & & & & & & & \\
\hline & & & Baseline & \multicolumn{2}{|c|}{ Follow-Up } & \multirow{2}{*}{$\begin{array}{l}\text { Baseline } \\
2016\end{array}$} & \multicolumn{2}{|c|}{ Follow-Up } & $\beta(D E)$ & \\
\hline & \multirow[t]{2}{*}{2017} & \multirow[t]{2}{*}{2018} & 2016 & 2017 & 2018 & & 2017 & 2018 & 201720 & \\
\hline & & & $(N=40)$ & $\begin{array}{l}(\mathrm{N}= \\
32)\end{array}$ & $\begin{array}{l}(\mathrm{N}= \\
31)\end{array}$ & $(\mathrm{N}=38)$ & $\begin{array}{l}(\mathrm{N}= \\
42)\end{array}$ & $\begin{array}{l}(\mathrm{N}= \\
40)\end{array}$ & & \\
\hline $\begin{array}{l}\text { Sweetened baked } \\
\text { goods }\end{array}$ & $100 \%$ & $100 \%$ & $85.0 \%$ & $87.5 \%$ & $83.9 \%$ & $81.6 \%$ & $73.8 \%$ & $72.5 \%$ & $\begin{array}{l}.36 \\
(1.20)\end{array}$ & $\begin{array}{l}.17 \\
(1.23)\end{array}$ \\
\hline \multicolumn{11}{|c|}{ Available at Check-Out ${ }^{d}$} \\
\hline Fresh fruit & $100 \%$ & $0 \%$ & $22.5 \%$ & $12.5 \%$ & $22.6 \%$ & $21.1 \%$ & $21.4 \%$ & $15.4 \%$ & $\begin{array}{l}-.62 \\
(1.12)\end{array}$ & $\begin{array}{l}.94 \\
(1.21)\end{array}$ \\
\hline Fresh vegetables & $0 \%$ & $0 \%$ & $2.5 \%$ & $0 \%$ & $0 \%$ & $2.6 \%$ & $0 \%$ & $0 \%$ & $\mathrm{n} / \mathrm{a}$ & $\mathrm{n} / \mathrm{a}$ \\
\hline Unflavored-milk & $0 \%$ & $0 \%$ & $2.5 \%$ & $0 \%$ & $0 \%$ & $0 \%$ & $0 \%$ & $0 \%$ & $\mathrm{n} / \mathrm{a}$ & $\mathrm{n} / \mathrm{a}$ \\
\hline Plain water & $100 \%$ & $0 \%$ & $2.6 \%$ & $6.3 \%$ & $6.5 \%$ & $2.6 \%$ & $7.1 \%$ & $5.1 \%$ & $\begin{array}{l}-.62 \\
(2.02)\end{array}$ & $\begin{array}{l}-.09 \\
(2.04)\end{array}$ \\
\hline Regular soda & $0 \%$ & $0 \%$ & $2.5 \%$ & $3.1 \%$ & $6.5 \%$ & $10.5 \%$ & $9.5 \%$ & $12.8 \%$ & $\mathrm{n} / \mathrm{a}$ & $\mathrm{n} / \mathrm{a}$ \\
\hline Diet soda & $0 \%$ & $0 \%$ & $0 \%$ & $3.1 \%$ & $3.2 \%$ & $7.9 \%$ & $7.1 \%$ & $7.7 \%$ & $\mathrm{n} / \mathrm{a}$ & $\mathrm{n} / \mathrm{a}$ \\
\hline $\begin{array}{l}\text { Other sweetened } \\
\text { beverages }\end{array}$ & $100 \%$ & $100 \%$ & $10.0 \%$ & $15.6 \%$ & $6.5 \%$ & $13.2 \%$ & $23.8 \%$ & $18.0 \%$ & $\begin{array}{l}-.68 \\
(1.58)\end{array}$ & $\begin{array}{l}-2.34 \\
(1.57)\end{array}$ \\
\hline Salty snacks & $0 \%$ & $0 \%$ & $37.5 \%$ & $71.9 \%$ & $51.6 \%$ & $55.3 \%$ & $45.2 \%$ & $57.5 \%$ & $\begin{array}{l}2.50 \\
(.97)^{\star}\end{array}$ & $.38(.94)$ \\
\hline Candy & $100 \%$ & $0 \%$ & $95.0 \%$ & $100 \%$ & $96.8 \%$ & $94.7 \%$ & $95.2 \%$ & $97.5 \%$ & $\mathrm{n} / \mathrm{a}$ & $\mathrm{n} / \mathrm{a}$ \\
\hline $\begin{array}{l}\text { Sweetened baked } \\
\text { goods }\end{array}$ & $100 \%$ & $0 \%$ & $42.5 \%$ & $59.4 \%$ & $45.2 \%$ & $50.0 \%$ & $45.2 \%$ & $60.0 \%$ & $\begin{array}{l}1.03 \\
(.90)\end{array}$ & $\begin{array}{l}-1.02 \\
(.97)\end{array}$ \\
\hline \multicolumn{11}{|c|}{ DID: Difference-In-Difference; SE: Standard Error. } \\
\hline \multicolumn{11}{|c|}{${ }^{*} \mathrm{p}$-value $<0.05 ;{ }^{* *} \mathrm{p}$-value $<0.01 ; * \star \star \mathrm{p}$-value $<0.001$} \\
\hline \multicolumn{11}{|c|}{ a.) Beta estimates and standard errors for intervention*time interaction term in differences-in-differences regression models. } \\
\hline \multicolumn{11}{|c|}{ b.) Exterior store ads include ads posted on the building and the store property. } \\
\hline \multicolumn{11}{|c|}{ c.) Interior store item displays includes special floor displays and end-aisle displays. } \\
\hline \multicolumn{11}{|c|}{ d.) Available at check-out includes items that are self-serve and clerk-assisted. } \\
\hline e.) DID model unak & duce $\mathrm{r}$ & Its. & & & & & & & & \\
\hline
\end{tabular}

\section{Discussion}

This quasi-experimental study aimed to evaluate the effect of a large chain supermarket opening in a predominantly low-income and African American community in Chicago, IL, on the existing retail food environment, which comprised mostly small stores that stock few staple food items. The overabundance of small food stores is particularly concerning because recent studies have found that excess availability of unhealthy food retailers (i.e., food swamp) is a better community-level predictor of obesity than poor availability of healthy food retailers (i.e., food desert) [23, 24]. Like other large urban centers in the U.S., many of Chicago's communities that are considered a food swamp or desert have a large percentage of poor and minority residents $[6,7,25]$. African American residents comprise $33 \%$ of Chicago's population but nearly $80 \%$ of the residents that live in a food desert [25]. Furthermore, the metropolitan Chicago area is one of the most segregated urban centers in the U.S. with high levels of black-white segregation [26]. Chicago's racial segregation has been linked to geographic inequities in health across the city [27, 28]. Since low- 
income African Americans are more at risk of chronic disease [29-31], addressing geographic disparities in healthy food access may narrow health inequities in Chicago.

The addition of a new supermarket to Englewood's retail food environment greatly expanded healthy food availability and promotion in the community. However, the entry of the supermarket did not lead to significant changes to food and beverage availability and marketing in nearby small food stores at one or two years after the opening. While statistically significant, changes to regular cheese availability (at one-year post intervention), salty snack availability at check-out (at one-year post intervention), and interior store promotion of other sweetened beverages (at two-years post-intervention) do not reflect a substantive change to the food landscape. These results underscore the hypothesis that small food stores in low resourced communities are not likely to alter their food and beverage stocking and marketing practices if a supermarket opens. Small food stores in these communities may fill a specific need of current residents, which keeps them economically viable despite new competition from a full-service grocer. A qualitative study by Sherman et al. (2015) highlighted the socio-cultural and economic significance of corners stores in low-income urban communities [32]. They reported that corner store usage often starts as a young age, and most use these stores to conveniently obtain discretionary items such as snacks and sugar-sweetened beverages [32]. Thus, the goods and services supplied by the supermarket may not supplant the primary motive for small food store usage by many community residents.

The findings from the current study are comparable to two other studies that recently evaluated the impact of introducing a HFFIsupported supermarket to a predominately low-income African American community [19, 20]. Singleton et al. (2019) reported that opening a discount supermarket, a Save-A-Lot, in a low-income area of Rockford, IL did not affect food and beverage availability and marketing in existing small food stores after one year post-opening $(N=22)$ [20]. Like the current study, many of the stores audited in Rockford were limited-service stores that stocked few staple food items [19]. Ghosh-Dastidar et al. (2017) conducted a natural experiment to examine how introducing a new supermarket to a low-income African American community in Pittsburgh, PA impacted healthy and unhealthy food availability and pricing in nearby stores $(\mathrm{N}=26)$ after a year [20]. They, too, found few changes in net food availability after the supermarket opened, although some changes in the pricing of certain food items were reported [19]. Economic studies provide some insight into this topic as well. For example, studies by Ailawadi et al. (2010) and Hausman and Leibtag (2007) documented how introducing a mass merchandiser (e.g., Wal-Mart or K-Mart) affected nearby retail stores $[17,18]$. While these studies linked the introduction of a mass merchandiser to loss of smaller discount retailers, they do not provide information on how these stores impact the existing retail food environment. Overall, the current state of evidence indicates that introducing a supermarket to a low-resourced urban community results in minimal change to food and beverage availability and marketing in existing small food stores. This evidence should be considered when putting the implications of large-scale healthy food retail expansion policies, such as the HFFI, into context.

Despite the increasing number of research studies on the $\operatorname{HFFI}[3,13,19,20,33-35]$, its effect on public health and healthy equity remains unclear. Expanding healthy food retail in low-resourced communities can potentially address major dietary concerns such as food insecurity and poor diet quality [33]. While the current study did not assess how the new supermarket impacted consumer food and beverage purchasing, food insecurity, or dietary intake in Englewood, a limited number of other studies have addressed this [35-38]. Overall, the evidence suggests that community perceptions of healthy food availability improve after a supermarket opens, but significant improvements in diet-related behaviors such as fruit and vegetable purchasing and consumption are not often observed [35-38]. Needed are studies that evaluate positive and negative changes to individual (e.g., food purchasing behavior, family feeding practices), community (i.e., social cohesion, blight), and other store-level factors (i.e., pricing, supply chains) in addition to food and beverage availability and marketing in smaller food stores [39]. The findings from studies that evaluated the impact of new mass merchandisers highlight an important concern: the possibly of business and residential displacement [16-18]. While it may generate jobs, the introduction of a high-end supermarket such as Whole Foods Market@ to a low-income minority community may also have unintended adverse consequences on the housing and financial security of currents residents. Recent studies on gentrification displacement have demonstrated both gradual and abrupt shifts in socio-demographics, housing value, and food retail outlet availability in response to new real estate investment projects [40-42]. This is a major concern that must be considered as future initiatives are introduced to improve the food landscape in low-income minority communities.

\section{Strengths and Limitations}


The strengths and limitations of the research should be noted. Using the Illinois PRC Food Store Observation Tool to audit stores was a strength because it features a large variety of food and beverage availability and marketing variables. The study design was a strength because a complete audit of all small food stores found in the intervention and comparison areas was performed at baseline and for two years follow-up. Nonetheless, the small sample size of small food stores found in the intervention and comparison areas may have affected the DID regression analyses, specifically the ability to detect significant differences between communities over time. A larger sample size of stores would have permitted a more robust assessment of change in food and beverage availability. Change in food and beverage pricing over time was not assessed in the current study. Given findings from similar studies that evaluated price, it is possible that existing small food stores in the current study altered their price of staple foods and snack items in response to the opening of the new supermarket [19]. Future studies should consider longitudinal changes to price and pricing strategies of healthy and unhealthy foods and beverages. No individual-level data were collected from community residents that allow an evaluation of changes in consumer food purchasing, decision-making, or dietary practices over time. Since this study was conducted solely in Chicago, findings may not be generalizable to other large urban centers in the U.S. or abroad.

\section{Conclusions}

Expanding healthy food retail in low-resourced communities has significant implications for public health and community nutrition because introducing a large chain supermarket may alter residents' food purchasing behaviors and perceptions about the healthfulness of the food landscape [35-38]. The current study observed no significant changes in food and beverage availability and marketing in Englewood's existing small food stores at one and two years after the introduction of a supermarket. However, the addition of this supermarket to the community's food landscape expanded the availability of healthy foods and beverages in an area of Chicago that previously lacked a supermarket for over a decade and has a large nutritionally vulnerable population. These findings contribute additional knowledge of the environmental and economic impact of healthy food retail initiatives such as the HFFI to the scientific literature. This information is integral for shaping future policy, systems, and environmental change interventions that aim to alleviate nutritional inequities in the U.S. Overall, there continues to be a need for studies that examine longitudinal changes to food pricing, food purchasing, dietary intake, and community stability in urban and rural settings after the introduction of a large full-service grocer. Future studies should address these gaps.

\section{Abbreviations}

DID (Difference-in-Difference); HFFI (Healthy Food Financing Initiative); SNAP (Supplemental Nutrition Assistance Program); USDA (United States Department of Agriculture)

\section{Declarations}

Ethnics Approval and Consent to Participate: The institutional review board that the University of Illinois Chicago deemed this exempt research.

\section{Consent for Publication: Not Applicable}

Availability of Data and Materials: The data collected and analyzed during the current study are available from the corresponding author on reasonable request.

Competing Interests: The authors declare that they have no competing interests.

Funding: The research presented in this paper was funded by the Centers for Disease Control and Prevention Nutrition and Obesity Policy Research and Evaluation Network (grant number: 5U48DP005010) and the National Cancer Institute of the National Institutes of Health (grant number R25CA057699).

Authors' Contributions: LP and SZ conceptualized the research project. CS participated in data collection and led the drafting of the manuscript. YL performed the data analysis. All authors read and approved the final manuscript. 
Acknowledgements: The authors would like to acknowledge the fieldworkers who participated in the data collection process, the storeowners and managers, and the Nutrition and Obesity Policy Research and Evaluation Network (NOPREN).

\section{References}

1. Office of Disease Prevention and Health

Office of Disease Prevention and Health. Promotion HP. 2020: Access to Foods that Support Healthy Eating Pattern. https://www.healthypeople.gov/2020/topics-objectives/topic/social-determinants-health/interventions-resources/access-tofoods-that (2020). Accessed 18 June 2020.

2. Hood C, Martinez-Donate A, Meinen A. Promoting Healthy Food Consumption: A Review of State-Level Policies to Improve Access to Fruits and Vegetables. WMJ. 2012;111:283-8.

3. Karpyn A, Young C, Weiss S. Reestablishing Healthy Food Retail: Changing the Landscape of Food Deserts. Child Obes. 2012;8:28-30.

4. Rose D. Access to Healthy Food: A Key Focus for Research on Domestic Food Insecurity. J Nutr. 2010;140:1167-9.

5. Vanderlee L, Olstad DL. Food Environment and Vulnerable Populations: Challenges and Opportunities for Policy. Health Promot Chronic Dis Prev Can. 2017;37:321-2.

6. Hilmers A, Hilmers DC, Dave J. Neighborhood disparities in access to healthy foods and their effects on environmental justice. Am J Public Health. 2012;10:1644-54.

7. Zenk SN, Powell LM, Rimkus L, et al. Relative and absolute availability of healthier food and beverage alternatives across communities in the United States. Am J Public Health. 2014;104:2170-8.

8. Reinvestment Fund. HFFI Bill Would Expand Healthy Food Access, Revitalize Communities. https://www.reinvestment.com/initiatives/hffi/ (2018). Accessed 18 June 2020.

9. U.S. Department of Health and Human Services, Office of Community Services. CED Healthy Food Financing Initiative FY 2016. https://www.acf.hhs.gov/ocs/programs/community-economic-development/healthy-food-financing (2019). Accessed 15 November 2019.

10. Ver Ploeg M, Nulph D, Williams R. Mapping Food Deserts in the United States. https://www.ers.usda.gov/amberwaves/2011/december/data-feature-mapping-food-deserts-in-the-us/ (2011). Accessed 13 March 2020.

11. Healthy Food Access.org. Illinois State \& Local Policy Efforts. https://www.healthyfoodaccess.org/illinois (2020). Accessed 18 June 2020.

12. Chicago Community Loan Fund. Whole Foods Market Celebrates Grand Opening in Englewood Square. https://cclfchicago.org/news/about-us/whole-foods-market-celebrates-grand-opening-in-englewood-square/ (2016). Accessed 10 January 2020.

13. Singleton CR, Li Y, Duran AC, Zenk SN, Odoms-Young A, Powell LM. Food and Beverage Availability in Small Food Stores Located in Healthy Food Financing Initiative Eligible Communities. Int J Environ Res Public Health. 2017;14:1-12.

14. Walker RE, Block J, Kawachi I. Do residents of food deserts express different food buying preferences compared to residents of food oases? A mixed-methods analysis. Int J Behav Nutr Phys Act. 2012;9:41.

15. Caspi CE, Lenk K, Pelletier JE, Barnes TL, Harnack L, Erickson DJ, et al. Association between store food environment and customer purchases in small grocery stores, gas-marts, pharmacies and dollar stores. Int J Behav Nutr Phys Act. 2017;14:76.

16. Jia P. What happens when Wal-Mart comes to town: an empirical analysis of the discount retailing industry. Econometrica. 2008;76:1263-316.

17. Hausman J, Leibtag E. Consumer benefits from increased competition in shopping outlets: measuring the effect of Wal-Mart. J Appl Econom. 2007;22:1157-77.

18. Ailawadi KL, Zhang J, Krishna A, Kruger MW. When Wal-Mart Enters: How Incumbent Retailers React and how this Affects their Sales Outcomes. J Market Res. 2010;47:577-93.

19. Ghosh-Dastidar M, Hunter G, Collins RL, Zenk S, Cummins S, Beckman R, et al. Does Opening a Supermarket in a Food Desert Change the Food Environment? Health Place. 2017;46:249-56.

Page $13 / 15$ 
20. Singleton CR, Li Yu, Odoms-Young A, Zenk SN, Powell LM. Change in Food and Beverage Availability and Marketing Following the Introduction of a Healthy Food Financing Initiative-Supported Supermarket. Am J Health Promot. 2019;33:525-33.

21. UIC Policy, Practice and Prevention Research Center. Tools. https://p3rc.uic.edu/wp-content/uploads/sites/561/2019/11/FinalNOPREN-instrument-11aug2015.pdf (2015). Accessed 24 June 2020.

22. Rimkus L, Powell LM, Zenk SN, Han E, Ohir-Vachaspati P, Pugach O, et al. Development and reliability testing of a food store observation form. J Nutr Educ Behav. 2013;45:540-8.

23. Kristen Cooksey-Stowers, Schwartz MB, Brownell KD. Food Swamps Predict Obesity Rates Better Than Food Deserts in the United States. J Environ Res Public Health. 2017;14:1366.

24. Hager ER, Cockerham A, O'Reilly N, Harrington D, Harding J, Hurley KM, et al. Food Swamps and Food Deserts in Baltimore City, MD, USA: Associations With Dietary Behaviours Among Urban Adolescent Girls. Public Health Nutr. 2017;20:2598-607.

25. Kolak M, Bradley M, Block DR, Pool L, Garg G, Toman CK, et al. Urban foodscape trends: Disparities in healthy food access in Chicago, 2007-2014. Health Place; 2018:52;231-9.

26. Henricks K, Lewis AE, Arenas I, Lewis DG. A Tale of Three Cities: The State of Racial Justice in Chicago Report. Institute for Research on Race and Public Policy. http://stateofracialjusticechicago.com/wpcontent/uploads/IRRPP_StateOfRacialJusticeReport-1.pdf (2017). Accessed 12 November 2019.

27. Mayne SL, Yellayi D, Pool LR, Grobman WA, Kershaw KN. Racial Residential Segregation and Hypertensive Disorder of Pregnancy Among Women in Chicago: Analysis of Electronic Health Record Data. Am J Hypertens. 2018;31:1221-7.

28. Tung EL, Hampton DA, Kolak M, Rogers SO, Yang JP, Peek ME. Race/Ethnicity and Geographic Access to Urban Trauma Care. JAMA. 2019;2:e190138.

29. Rehm CD, Peñalvo JL, Afshin A, Mozaffarian D. Dietary Intake Among US Adults, 1999-2012. JAMA. 2016;315:2542-53.

30. O'Keefe EB, Meltzer JP, Bethea TN. Health Disparities and Cancer: Racial Disparities in Cancer Mortality in the United States, 2000-2010. Front Public Health. 2015;3:51.

31. Krueger PM, Reither EN. Mind the Gap: Race\Ethnic and Socioeconomic Disparities in Obesity. Curr Diab Rep. 2015;15:95.

32. Sherman S, Grode G, McCoy T, Vander Veur SS, Wojtanowski A, Almaguer Sandoval B, et al. Corner Stores: The Perspective of Urban Youth. J Acad Nutr Diet. 2015;115:242-8.

33. Chrisinger B. Taking Stock of New Supermarkets in Food Deserts: Patterns in Development, Financing, and Health Promotion. https://www.ncbi.nlm.nih.gov/pmc/articles/PMC6296768/ (2016). Accessed 2 July 2020.

34. Dubowitz T, Ncube C, Leuschner K, Tharp-Gilliam S. A natural experiment opportunity in two low-income urban food desert communities: research design, community engagement methods, and baseline results. Health Educ Behav. 2015;42:87-6.

35. Dubowitz T, Ghosh-Dastidar M, Cohen DA, Beckman R, Steiner ED, Hunter GP, et al. Diet and perceptions change with supermarket introduction in a food desert, but not because of supermarket use. Health Aff. 2015;34:1858-68.

36. Chrisinger B. A mixed-methods assessment of a new supermarket in a food desert: contributions to everyday life and health. $J$ Urban Health. 2016;93:425-37.

37. Elbel B, Mijanovich T, Kiszko K, Abrams C, Cantor J, Dixon LB. The introduction of a supermarket via tax-credits in a low-income area. Am J Health Promot. 2017;31:59-66.

38. Rogus S, Athens L, Cantor J, Elbel B. Measuring micro-level effects of a new supermarket: do residents within 0.5 mile have improved dietary behaviors. J Acad Nutr Diet. 2017;118:1037-46.

39. Fleischhacker SE, Flournoy R, Moore LV. Meaningful, measurable, and manageable approaches to evaluating healthy food financing initiatives: an overview of resources and approaches. J Public Health Manag Pract. 2013;19:541-9.

40. Cohen N. Feeding or Starving Gentrification: The Role of Food Policy. https://static1.squarespace.com/static/572d0fcc2b8dde9e10ab59d4/t/5aba9936575d1fe8933df34e/1522178358593/PolicyBrief-Feeding-or-Starving-Gentrification-20180327-Final.pdf (2018). Accessed 2 July 2020.

41. Whittle HJ, Palar K, Hufstedler LL, Seligman HK, Frongillo. Weiser EA. SD.

42. Food insecurity. chronic illness, and gentrification in the San Francisco Bay Area: An example of structural violence in United States public policy. Soc Sci Med. 2015;143:451-61. 
43. Alkon AH, Cadji J. Sowing Seeds of Displacement: Gentrification and Food Justice in Oakland, CA. Int J Urban Reg Res. 2018;44:108-23. 